\title{
SUMOylation of PES1 upregulates its stability and function via inhibiting its ubiquitination
}

\author{
Shujing $\mathrm{Li}^{1}$, Miao Wang ${ }^{1}$, Xinjian $Q \mathbf{u}^{2}$, Zhaowei $\mathrm{Xu}^{1}$, Yangyang Yang ${ }^{1}$, Qiming Su${ }^{2}$, \\ Huijian $\mathbf{W u}^{1,2}$ \\ ${ }^{1}$ School of Life Science and Biotechnology, Dalian University of Technology, Dalian, China \\ ${ }^{2}$ School of Life Science and Medicine, Dalian University of Technology, Panjin, China \\ Correspondence to: Huijian Wu, email: wuhj@dlut.edu.cn
}

Keywords: PES 1, SUMOylation, breast cancer, ubiquitination

Received: July 21, $2015 \quad$ Accepted: June 15, $2016 \quad$ Published: July 08, 2016

\section{ABSTRACT}

PES1 is a component of the PeBoW complex, which is required for the maturation of 285 and 5.85 ribosomal RNAs, as well as for the formation of the 605 ribosome. Deregulation of ribosomal biogenesis can contribute to carcinogenesis. In this study, we showed that PES1 could be modified by the small ubiquitin-like modifier (SUMO) SUMO-1, SUMO-2 and SUMO-3, and SUMOylation of PES1 was stimulated by estrogen (E2). One major SUMOylation site (K517) was identified in the C-terminal Glu-rich domain of PES1. Substitution of $\mathrm{K517}$ with arginine abolished the SUMOylation of PES1. SUMOylation also stabilized PES1 through inhibiting its ubiquitination. In addition, PES1 SUMOylation positively regulated the estrogen signaling pathway. SUMOylation enhanced the ability of PES1 to promote estrogen receptor a (ERa)mediated transcription by increasing the stability of ERa, both in the presence and absence of E2. Moreover, SUMOylation of PES1 also increased the proportion of $S$-phase cells in the cell cycle and promoted the proliferation of breast cancer cells both in vitro and in vivo. These findings showed that posttranslational modification of PES1 by SUMOylation may serve as a key factor that regulates the function of PES1 in vivo.

\section{INTRODUCTION}

PES1 is a nuclear protein discovered in 1996, and early studies have shown that several abnormal developments that occurred in the brain, eye, liver and other organs of zebrafish embryo as a result of PES1 mutation can even cause the death of the embryo $[1,2]$. The human PESI gene, which is located to $22 \mathrm{q} 12.1$ was first cloned in 2001 [3]. The PES1 protein contains 588 amino acids and it has three nuclear localization signals (NLSs) that mediate the transport of the protein into the nucleus. PES1 is mainly localized in the nucleolus, and rarely distributed in the cytoplasm. The $\mathrm{C}$ terminus (amino acids 315-412) of PES1 contains a typical BRCT domain that has been found in the carboxyl terminus of one breast cancer suppressor protein, BRCA1. Many proteins containing the BRCT domain play a role in DNA repair, remodeling and cell cycle. The BRCT domain of PES1 plays a crucial role in regulating the progress of cell cycle [4]. In addition, PES1 also contains a glutamate rich region, but its function is not clear.
The main function of PES1 is its involvement in the synthesis and maturation of ribosome and its effect on chromatin stretch. More and more research have shown that PES1 is closely associated with tumorgenesis via improving cell proliferation and participating in the progress of cell cycle in cancer cells. PES1 is mainly expressed in developing tissues, but aberrant expression of PES1 has been found in human brain tumors. Neuroblastoma (NB) cases with MYCN amplification and international neuroblastoma staging system stage 4 (INSS stage 4) tend to show a higher expression level of PES1 [5]. In addition, high PES1 expression is associated with the worst overall and relapse-free survival [5]. PES1 is also upregulated in several different carcinoma cell lines, such as the colon cancer cell line SW480 and human breast cancer cell line MCF-7 [6]. PES1 is upregulated more in colon cancer tissues than in non-cancerous tissues, while down-regulation of PES1 in colon cancer cells can lead to cell cycle arrest at the G1 phase, as well as reduced proliferation and decreased growth of xenografts [7]. Clinical study has suggested that the expression of PES1 
in breast cancer tissues (stage I-IV is much higher than that in normal breast tissues, while PES1 knockdown represses the growth and tumorgenesis of breast cancer cells [8]. One possible mechanism by which PES1 promotes the development of breast cancer (ER $\alpha$-positive) is through increasing the level of ER $\alpha$ while decreasing the level of $\mathrm{ER} \beta$ [9]. ER $\alpha$ promotes the stimulating effect of estrogen on breast cancer cell proliferation, whereas ER $\beta$ inhibits the proliferation of breast cancer cells. All these data show that abnormality associated with PES1 may be a common feature of malignancy.

Small ubiquitin like-modifier (SUMO) modification is a crucial post-translational modification for regulating protein activity, stability, alteration of protein-protein interaction and transcriptional activity as well as protein sublocalization in the cells. A consensus sequence $\Psi \mathrm{KXE}$ exists in most proteins that are modified by SUMOylation. In this sequence, $\Psi$ stands for a hydrophobic amino acid and $\mathrm{K}$ is the conjugation site for SUMO. SUMOylation modification is a dynamic process, in which the modified proteins can be deSUMOylated by SUMO-specific proteases (SENPs) [10]. Many proteins with important roles in cellular processes have been identified as targets that are modified by SUMO. For examples, SUMOylation of DEC1 increases its ability to repress the transcriptional activity of CLOCK/BMAL1 [11], and SUMOylation of GPS2 promotes its ability to inhibit ER $\alpha$-mediated transcription [12]. Sequence analysis of PES1 revealed two potential SUMO conjugation sites, K249 and K517, both of which were contained within the consensus sequence $\Psi \mathrm{KXE}$, so we wanted to examine whether PES1 is a target protein of SUMO.

In this study, we demonstrated that PES1 could be SUMOylated by SUMO-1, -2 and -3 , and showed that K517 was the main SUMOylation site of PES1. SUMOylation stabilized PES1 byinhibiting its ubiquitination. PES1 SUMOylation also promoted the transcriptional activity of $\mathrm{ER} \alpha$ by enhancing its stability. SUMOylation of PES1 inhibited the association of ER $\alpha$ with its ubiquitination E3 ligase ChIP and as well as inhibiting the ubiquitination of $\mathrm{ER} \alpha$. Moreover, SUMOylation of PES1 increased the percentage of MCF7 cells in the S phase and promoted the proliferation of breast cancer cells as well as the formation of xenograft tumor. Taken together, our data suggested that posttranslation modification of PES1 via SUMOylation might play important roles in ER $\alpha$-related estrogen signaling pathway and in the growth of breast cancer cells.

\section{RESULTS}

\section{PES1 is modified by SUMO}

To identify whether PES1 is a SUMOylated substrate, COS-7 cells were cotransfected with GFPPES1 and Myc-SUMO-1, -2 or -3 . Western blot analysis of PES1 showed a band with a molecular mass much higher than that of PES1 when PES1 was coexpressed with SUMO-1, -2 or -3 (Figure 1A), suggesting that PES1 was modified by all three kinds of SUMO. Since PES1 was mainly modified by SUMO-1, we therefore focused mainly on the functions of PES1 modified by SUMO-1 in subsequent experiments. Moreover, analysis of PES1 SUMOylation in COS-7 cells cotransfected with FlagPES1 and Myc-SUMO-1 showed that the overexpressed PES1 was modified by SUMO-1 (Figure 1B). To further confirm the SUMOylation of PES1, we cotransfected COS-7 cells with Flag-PES1 and GFP-SUMO-1 or GFPSUMO-1 GA and analyzed the SUMOylated products. SUMO-1 GA is a SUMO mutant that lacks the ability to join with the substrate due to a C-terminal diglycine substitution (GG to GA). A band of higher molecular mass indicative of PES1-SUMO1 was detected only when PES1 was coexpressed with wild-type SUMO-1 in the cells (Figure 1C). The effect of UBC9, a SUMO E2 conjugate enzyme, on the SUMOylation of PES1 was also examined. Cells transfected with UBC9 and SUMO-1 showed the highest intensity for the PES1-SUMO-1 band (Figure 1D). In addition, a SUMOylation assay conducted with purified recombinant SUMO-conjugating enzymes and GST-PES1 clearly showed that PES1 could be modified by SUMO-1 in vitro (Figure 1E). The effect of estrogen (E2) on endogenous SUMOylated PES1 was also investigated. PES1 immunoprecipitated from MCF-7 cells was detected by anti-SUMO1 antibody, and its level increased when the cells were treated with E2 (Figure 1F). To further confirm this result, MCF-7 cells were transfected with Myc-SUMO-1, and PES1 was then immunoprecipitated from the cells and probed with anti-Myc antibody. The result showed that the intensity of the band corresponding to SUMOylated PES1 increased with E2 treatment (Figure $1 G)$. The protein level of PES1 was upregulated when the E2 treatment lasted for $8 \mathrm{~h}$, but remained unchanged when the E2 treatment lasted for $4 \mathrm{~h}$ (Figure $1 \mathrm{H})$. These data suggested that PES1 could be modified by SUMO, and E2 may upregulate the SUMOylation of PES1.

\section{K517 is the primary site for PES1 SUMOylation}

Analysis of the sequence of PES1 revealed two conserved lysine residues in the Glu-rich domain: one corresponding to K249 and the other to K517 (Figure 2A). Furthermore, these two sites are conserved in different species (homo, mouse, marmoset and xenopus) (Figure 2A). Changing K249 to arginine (K249R) resulted in nearly no change in the SUMOylation status of PES1, whereas changing K517 to arginine (K517R) or both K249 and K517 to arginine (2KR) appeared to abolish the SUMOylation of PES1 (Figure 2B). To further define the SUMOylation sites in PES1, COS-7 cells were transfected with Myc-SUMO1 together with GFPPES1 or GFP-K517R. GFP-PES1 immunoprecipitated 
from the cell extract was detected by anti-Myc antibody, while the immunoprecipitated GFP-K517R did not show any reaction with the anti-Myc antibody, indicating that the SUMO-1 did not conjugate with the mutant PES1 (Figure 2C). The effect of E2 on the SUMOylation of the mutant PES1 was also examined. E2 slightly increased the SUMOylation of wild-type PES1, but had no effect on the SUMOylation of the mutant PES1 (Figure 2D). These data indicated that K517 of PES1 could be the key SUMOylation site.

\section{Effect of SUMOylation on the localization and stability of PES1}

As reported in previous study [13], PES1 is localized in the nuclei of AR5 cells (human fibroblast cell line). SUMOylation is considered to influence the functions of proteins by changing their properties such as subcellular localization and protein stability. We speculated that SUMOylation might affect the subcellular localization of PES1. To test this

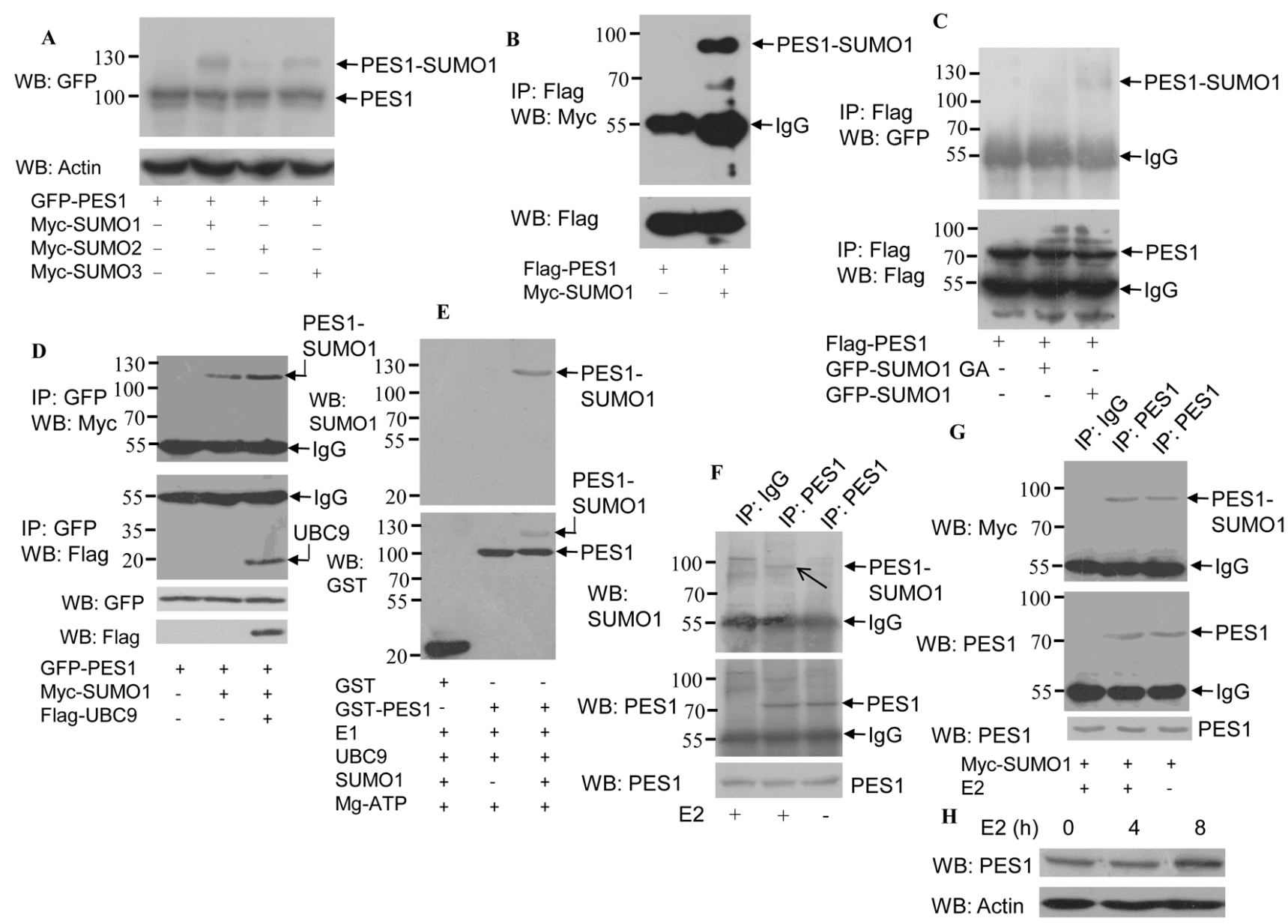

Figure 1: Modification of PES1 by SUMO-1, SUMO-2 and SUMO-3. A. COS-7 cells were co-transfected with Myc-SUMO-1, -2 or -3 and GPF-tagged PES1, and then subjected to western blot with anti-GFP antibody. B. COS-7 cells were transfected with Flagtagged PES1 and Myc-tagged SUMO1 and then subjected to immunoprecipitation with anti-Flag antibody followed by western blot with anti-Myc antibody. C. COS-7 cells were cotransfected with Flag-tagged PES1 and GFP-tagged SUMO1 or SUMO1/GA and then subjected to immunoprecipitation with anti-Flag antibody, followed by western blot with anti-GFP antibody. D. COS-7 cells were transfected with GFP-PES1 alone, GFP-PES1 plus Myc-SUMO1 without or with UBC9, and then subjected to immunoprecipitation with anti-GFP antibody followed by western blot with anti-Myc or anti-Flag antibody. E. In vitro SUMOylation assay was performed using a SUMOylation kit (Enzo Life Sciences). Affinity-purified GST or GST-PES1 was incubated with SAE1-SAE2 (E1), UBC9 (E2), Mg-ATP and SUMO-1, as indicated at $30^{\circ} \mathrm{C}$ for $60 \mathrm{~min}$, and then analyzed by immunoblotting. F. MCF-7 cells were subjected to serum starvation for 3 days, followed by treatment with $10 \mathrm{nM} \mathrm{E} 2$ for $4 \mathrm{~h}$, and the cell extract was then subjected to immunoprecipitation with anti-PES1 antibody or anti-immunoglobulin G, followed by western blot analysis with anti-SUMO1 or anti-PES1 antibody. G. MCF-7 cells were transfected with Myc-SUMO1 for $24 \mathrm{~h}$, and the cells were then subjected to serum starvation for 3 days, followed by treatment with $10 \mathrm{nM}$ E2 for $4 \mathrm{~h}$. Cell extract was prepared and subjected to immunoprecipitation with anti-PES1 antibody or anti-immunoglobulin G, followed by western blot analysis with anti-Myc or anti-PES1 antibody. H. MCF-7 cells were starved for 3 days, followed by treatment with $10 \mathrm{nM}$ E2 for different periods, and then subjected to western blot analysis with anti-PES1 antibody. 
speculation, we transfected MCF-7 cells with GFPtagged PES1 or its mutant K517R and the cells were then subjected to immunofluorescence assay. The result showed that PES1 was mainly localized in the nucleus of the cells, regardless of whether it was wildtype or mutant PES1 (Figure 3A). This indicated that SUMOylation might not influence the subcellular localization of PES1.

The effect of SUMOylation on PES1 protein stability was examined by the half-life assay. The half-life of wild-type PES1 was more than $8 \mathrm{~h}$, while the half-life of the mutant was between $6 \mathrm{~h}$ and $8 \mathrm{~h}$ (Figure 3B). This indicated that SUMOylation stabilized the protein level of PES1. Since the stability of a protein may be subject to regulation by ubiquitination, it became necessary to examine whether the increased stability of SUMOylated PES1 was due to suppression of its ubiquitination. Ubiquitination wild-type PES1 was reduced compared to the mutant (Figure 3C), showing that SUMOylation of PES1 repressed its ubiquitination.

\section{PES1 K517R promotes Trim23-mediated PES1 ubiquitin-proteasome pathway}

To further study the role of SUMOylation in the regulation of PES1 stability, the effects of several ubiquitin E3 ligases were investigated. PES1 level was most significantly affected by Trim 23 (data not shown). COS-7 cells transfected with Trim 23 plus GFP-tagged wild-type PES1 or mutant PES1 (K517R) showed reduced protein stability with respect to PES1, but the level of K517R was much lower than that of wild type (Figure 4A). Co-immunoprecipitation showed that the interaction between wild-type PES1 and Trim23 was noticeably weaker than the interaction between K517R and Trim23 (Figure 4B). In addition, the ubiquitination of PES1 K517R was increased compared to that of wild-type PES1, especially in the presence of Trim23 (Figure 4C). On the other hand, the ubiquitination of PES1 was decreased when Trim23 was knockdown (Figure 4E) (Si Trim23-1377 appeared to be more effective than the other two shown in Figure 4D). The protein level of PES1 (both wild type and K517R) was
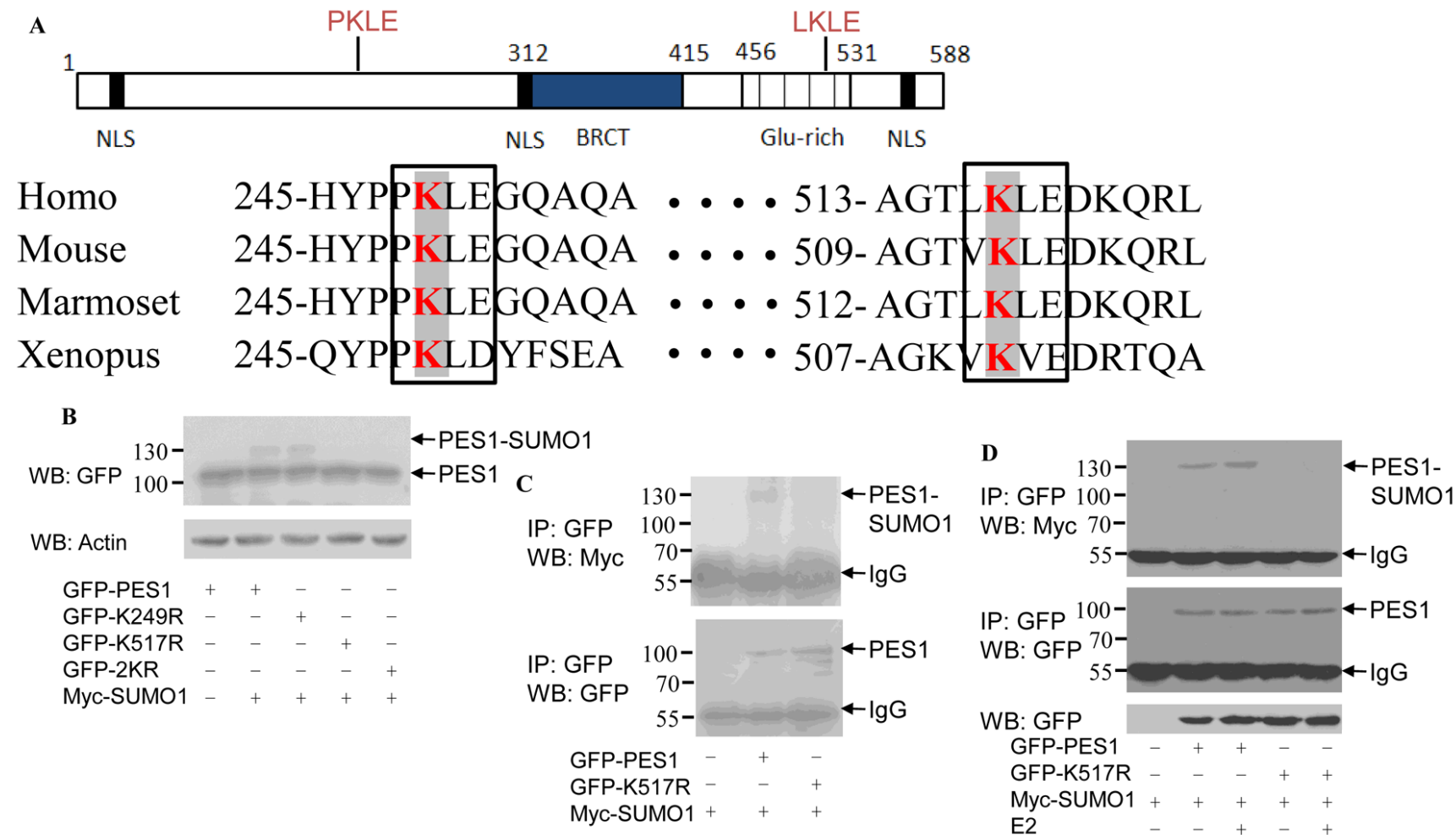

Figure 2: Identification of SUMOylation sites in PES1. A. Schematic representation of human PES1, showing the conserved lysine residues within the Glu rich region. The amino acids surrounding the two conserved lysine residues were compared with those of PES1 from three other species: mouse, Marmoset and Xenopus. Conserved lysine residues are boxed. B. COS-7 cells were transfected with wild-type GFP-PES1 or its mutants K249R, K517R or K249R/K517R (2KR) and Myc-tagged SUMO-1, and then subjected to western blot with anti-GFP antibody. C. COS-7 cells were transfected with wild-type GFP-PES1 or its mutant K517R as well as Myc-SUMO-1, and then subjected to immunoprecipitation with anti-GFP antibody followed by western blot with anti-Myc or anti-GFP antibody. D. MCF-7 cells were transfected with wild-type GFP-PES1 or its mutant K517R as well as Myc-SUMO-1 for 24 h, and the cells were then subjected to serum starvation for 3 days, followed by treatment with $10 \mathrm{nM}$ E2 for $4 \mathrm{~h}$. Cell extract was then prepared and subjected to immunoprecipitation with anti-GFP antibody, followed by western blot analysis with anti-Myc or anti-GFP antibody. 
also increased with the knockdown of Trim23 (Figure 4F). Taken together, the results indicated that SUMOylation of PES1 may inhibit its interaction with Trim23, resulting in increased stability for PES1.

\section{Effect of SUMOylation on protein stability of ERa}

PES1 promotes the growth of breast cancer cells by improving the stability of ER $\alpha$ [9]. Overexpression of wildtype PES1 in COS-7 cells increased the protein level of $\mathrm{ER} \alpha$ while overexpression of K517R caused no change to the level of ER $\alpha$ (Figure 5A). PES1 promotes the stability of the $\mathrm{ER} \alpha$ through inhibiting the interaction between $\mathrm{ER} \alpha$ and the carboxyl terminus of Hsc70-interacting protein (CHIP), and subsequently repressing the ubiquitinproteasome pathway-mediated degradation of ER $\alpha$ [14]. Next we examined whether PES1 SUMOylation plays a role in regulating the interaction between $\mathrm{ER} \alpha$ and CHIP. Interaction between ER $\alpha$ and CHIP was not detected in the extract of the cells that overexpressed wild-type PES1, but was detected in the extract of the cells that overexpressed the mutant PES1 or did not overexpress PES1 (Figure 5B). This data demonstrated that SUMOylation of PES1 inhibited the interaction between CHIP and ER $\alpha$. Moreover, the role of
PES1 SUMOylation in the ubiquitination of ER $\alpha$ was also examined. Overexpression of wild-type PES1 decreased the ubiquitination of ER $\alpha$ compared to the control (no overexpression of PES1), while the mutant PES1 K517R had a weaker effect on the ubiquitination of ER $\alpha$ compared to wild-type PES1 (Figure 5C). In addition, PES1-mediated ER $\alpha$ stability was not affected by E2, although wild-type PES1 conferred a higher degree of stability to ER $\alpha$ than K517R. (Figure 5D and 5E). Overexpression of UBC9 together with wild-type PES1 further promoted the stability of ER $\alpha$ (Figure 5F). These data showed that the stability of ER $\alpha$ promoted by PES1 was dependent on its SUMOylation.

\section{SUMOylation of PES1 promoted the transcriptional activity of ER $\alpha$}

To examine whether SUMOylation of PES1 also affected the transcriptional activity of $\operatorname{ER} \alpha$, luciferase reporter assays were performed. As anticipated, the highest level of luciferase activity was found in cells that overexpressed both ER $\alpha$ and wild-type PES1, whether with or without E2 treatment, while overexpression of $\mathrm{K} 517 \mathrm{R}$ reduced its ability to activate the transcriptional activity of ER $\alpha$ (Figure 6A). The effect of PES1
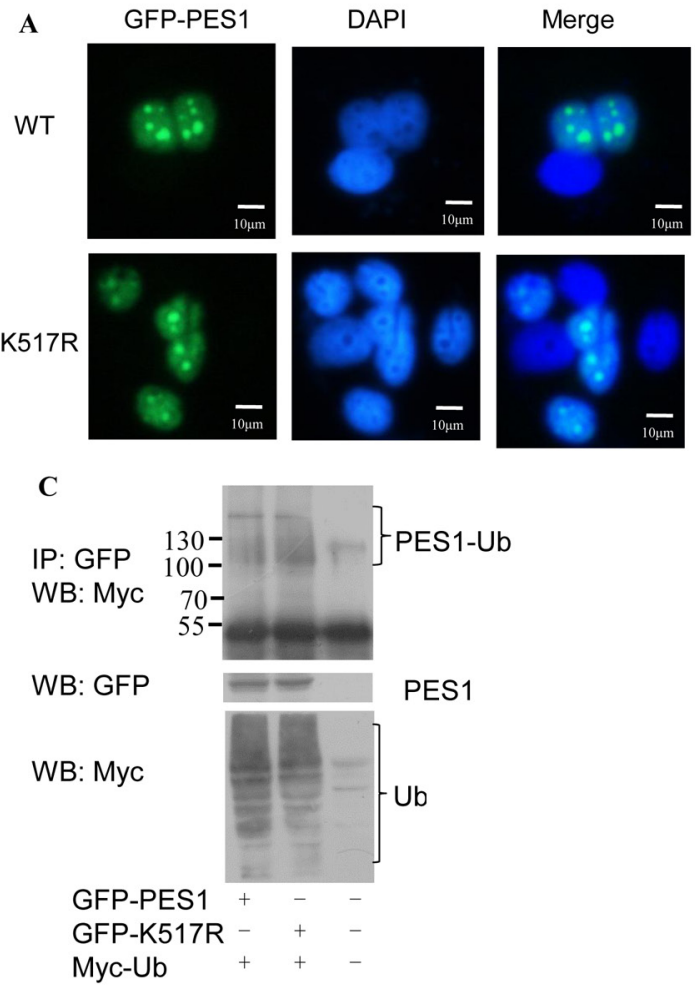

B

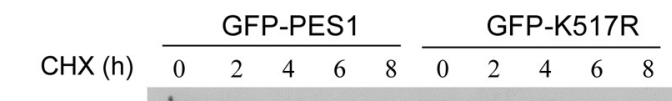

WB: GFP
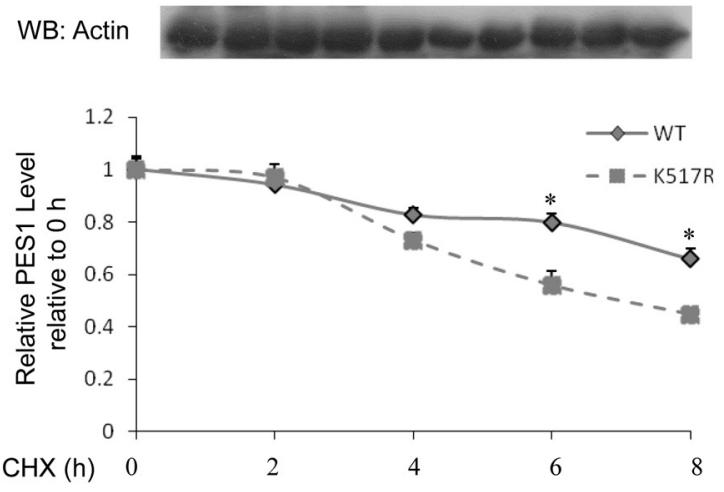

Figure 3: Effect of SUMOylation on the nucleolar distribution and stability of PES1. A. MCF-7 cells transfected with GFPtagged wild-type PES1 or mutant PES1 K517R were stained with GFP. Nuclei were stained with 4,6-diamidino-2-phenylindole (DAPI). B. MCF-7 cells were transfected with GFP-tagged wild-type PES1 or K517R and treated with CHX at the indicated time periods. Cell lysate was subjected to western blot with anti-GFP antibody. C. MCF-7 cells were transfected with GFP-tagged wild-type PES1 or K517R together with Myc-tagged ubiquitin, and then treated with MG132, a proteasome inhibitor, for $6 \mathrm{~h}$ before harvest. Cell lysate was subjected to immunoprecipitation with anti-GFP antibody followed by western blot with anti-Myc antibody. 
SUMOylation on the regulation of cyclin D1 expression, a well established ER $\alpha$-target gene, was also examined. Wild-type PES1 promoted the expression of cyclin D1 in the presence or absence of E2, but this effect of PES1 was compromised when the SUMOylation site was mutated (Figure 6B). Overexpression of UBC9 together with wild-type PES1 further promoted the activity of ERE-Luc reporter in MCF-7 and T47D cells (Figure 6C). Similar result was also obtained from RT-PCR analysis, where a higher level of cyclin D1 mRNA was found in the cells transfected with wild-type PES1 than in the cells transfected with the mutant PES1 (Figure 6D). All these data showed that PES1 SUMOylation had a crucial effect on the regulation of $\mathrm{ER} \alpha$ activity.

\section{Effect of PES1 SUMOylation on breast cancer cell lines proliferation}

The role of PES1 SUMOylation in cell proliferation was investigated by determining the effect of PES1 SUMOylation on the proliferation of two breast cancer cell lines. MCF-7 cells overexpressing wild-type PES1 showed more proliferation than those overexpressing K517R, and similar result was observed for T47D cells (Figure 7A). Furthermore, as demonstrated with MCF-7 cells, a greater number of cells appeared when the cells overexpressed wildtype PES1 compared to those that overexpressed K517R (Figure 7B). We next examined the effect of wild-type PES1 or K517R on the colony formation of MCF-7 cells.
A

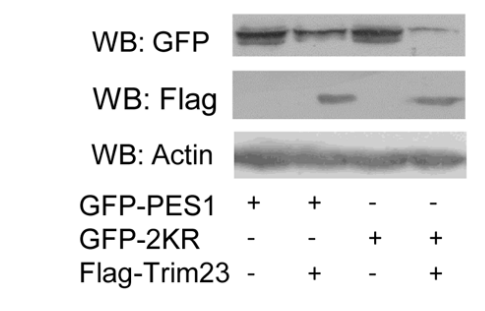

D

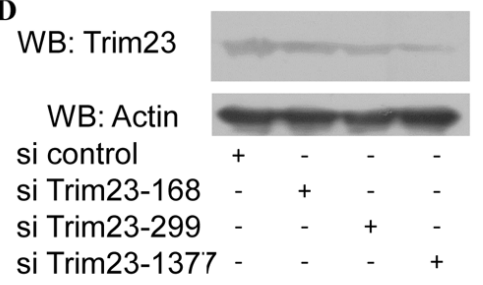

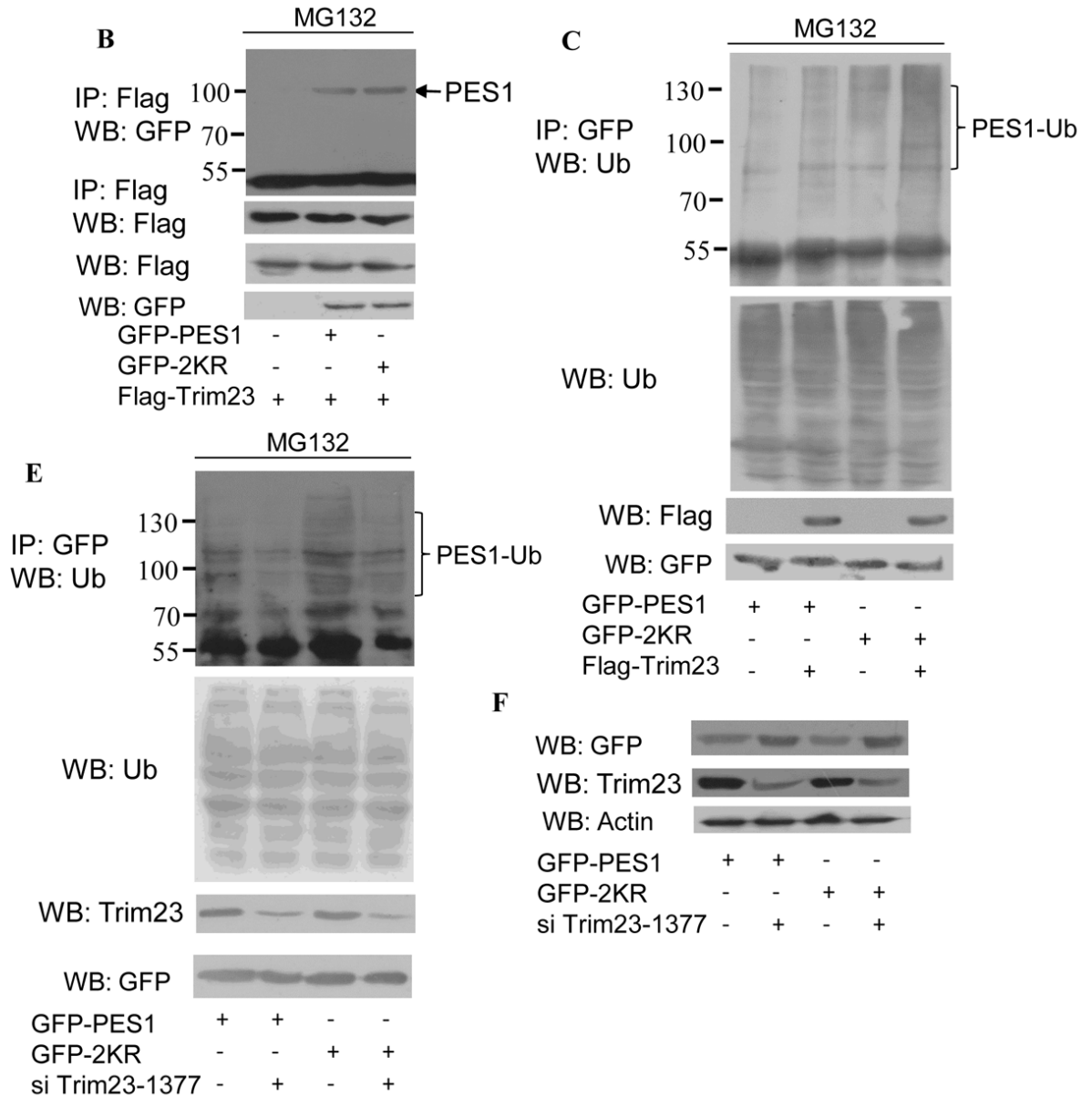

Figure 4: Effect of ubiquitin E3 ligase Trim23 on the stability of PES1. A. COS-7 cells were transfected with GFP-tagged wildtype PES1 or mutant PES1 K517R together with Flag-Trim23 or not, and then subjected to western blot with anti-GFP antibody. B. COS-7 cells were transfected with Flag-tagged Trim23 plus GFP-tagged wild-type PES1 or K517R, and then treated with MG132, a proteasome inhibitor, for $6 \mathrm{~h}$ before harvest. Cell lysate was subjected to immunoprecipitation with anti-Flag antibody followed by western blot with anti-GFP antibody or anti-Flag antibodies. C. COS-7 cells were transfected with GFP-tagged wild-type PES1 or K517R without or with Trim23, and then treated with MG132, a proteasome inhibitor, for $6 \mathrm{~h}$ before harvest. Cell lysate was subjected to immunoprecipitation with anti-GFP antibody followed by western blot with anti-ubiquitin antibody. D. MCF-7 cells transfected with si control, si Trim23-168, si Trim23-299 or si Trim23-1377 were subjected to western blot with anti-Trim23 antibody. E. MCF-7 cells were transfected with GFPPES1 or GFP-PES1 K517R together with or without si-Trim23-1377. The cell extract was subjected to immunoprecipitation with anti-GFP antibody, followed by western blot using anti-Ub antibody. F. MCF-7 cells were transfected with GFP-PES1 or GFP-PES1 K517R together with or without si-Trim23-1377, and then subjected to western blot with anti-GFP antibody. 
MCF-7 cells overexpressed GFP-tagged wild-type PES1 showed more proliferation than those that either did not express PES1 or overexpressed K517R (Figure 7C). The effect of PES1 SUMOylation on the process of cell-cycle was also studied. MCF-7 cells that overexpressed wild-type PES1showedan overall increase in the proportion of S-phase cells, with a corresponding decrease in the proportion of cells at the G0/G1 phase compared to the control cells (no overexpression of PES1) (Figure 7D). The proportion of cells in the S-phase also increased when the cells overexpressed PES1 K517R, compared to control cells, but to a lesser extent than those overexpressed wild-type PES1 (Figure 7D). Thus K517R appeared to have a lesser effect on the cell cycle. These data demonstrated that SUMOylation of PES1 could promote the proliferation of breast cancer cells. Overexpression of UBC9 together with wild-type PES1 further promoted the growth of both breast cancer cell lines (Figure 7E). To further identify the role of PES1 SUMOylation in tumorigenesis in vivo, nude mice were subcutaneously implanted with MCF-7 cells that stably overexpressed GFP-tagged PES1, PES1 K517R or GFP-C1. Mice implanted with MCF-7 cells that overexpressed GFP-tagged PES1 showed a much larger tumor than mice implanted with MCF-7 cells that overexpressed PES1 mutant or just harbored the control vector during the experimental period (Figure 7F and 7G). Forty four days after the implantation of tumor cells, a 3-fold increase in the weight of the tumor was observed for MCF-7 cells that overexpressed wild-type PES1 compared to tumors derived from cells carried the control vector (Figure 7H). Western blot analysis of the tumors showed that PES1 was successfully expressed (Figure 7I). Overall, these data indicated that wild-type PES1 could promote the growth of cancer cells in vitro and in vivo.

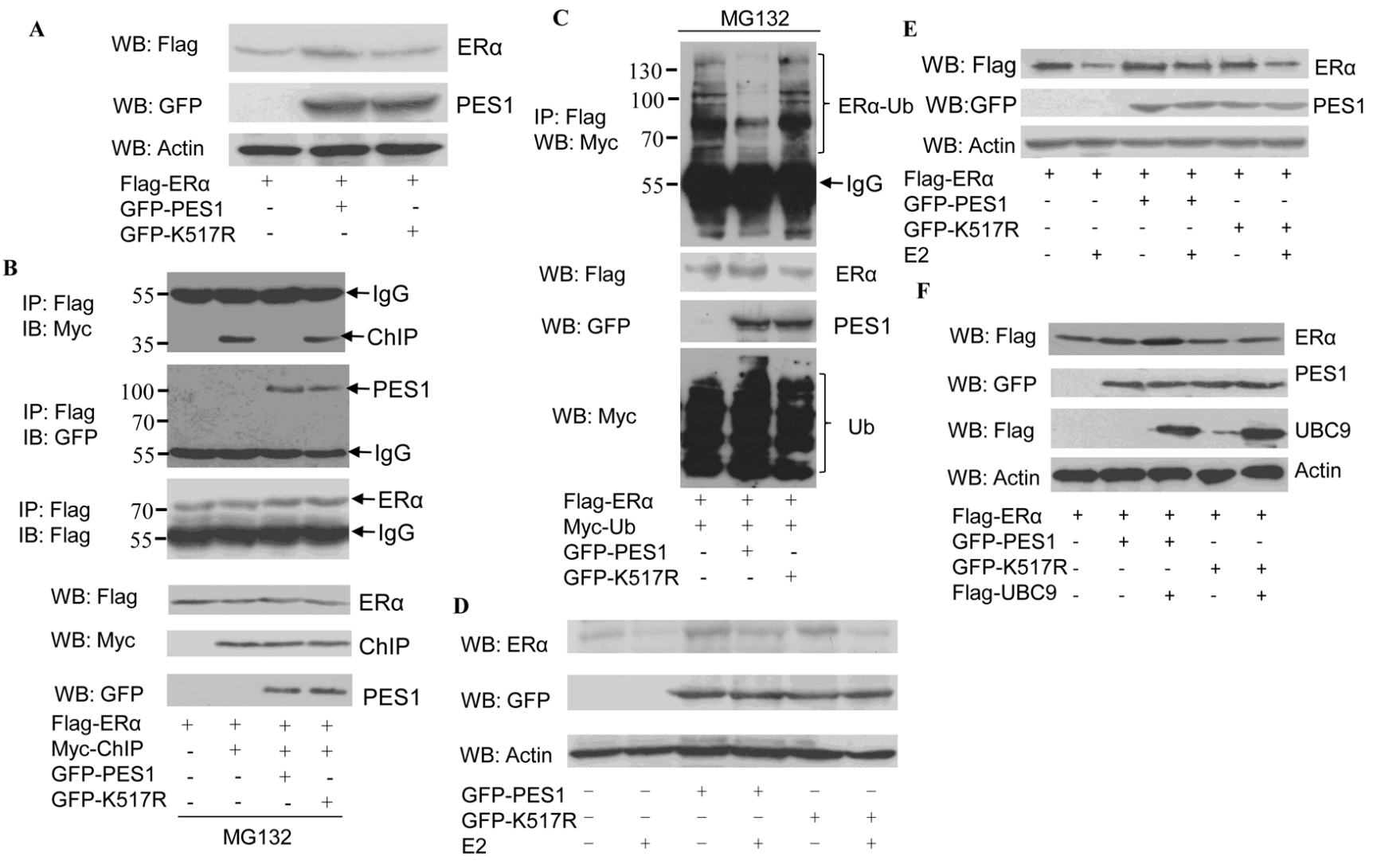

Figure 5: Effect of PES1 SUMOylation on the stability of ER $\boldsymbol{\alpha}$. A. COS-7 cells were transfected with Flag-ER $\alpha$ together with GFP-tagged wild-type PES1 or K517R, and then subjected to western blot with anti-Flag antibody. B. COS-7 cells were transfected with Flag-ER $\alpha$ and control vector or Myc-ChIP together with GFP-tagged wild-type PES1 or K517R, and then treated with MG132, a proteasome inhibitor, for $6 \mathrm{~h}$ before harvest. Cell lysate was subjected to immunoprecipitation with anti-Flag antibody followed by western blot with anti-Myc, anti-GFP or anti-Flag antibodies. C. COS-7 cells were transfected with Flag-ER $\alpha$ and Myc-Ub together with GFP-tagged wildtype PES1 or K517R, and then treated with MG132 for $6 \mathrm{~h}$ before harvest. Cell lysate was subjected to immunoprecipitation with anti-GFP antibody followed by western blot with anti-Myc antibody. D. MCF-7 cells were transfected with GFP-tagged wild-type PES1 or K517R for $24 \mathrm{~h}$. The cells were starved for 3 days, and then treated with or without $10 \mathrm{nM} \mathrm{E} 2$ for $24 \mathrm{~h}$. The cell extract was subjected to western blot with anti-ER $\alpha$. E. MCF-7 cells were transfected with Flag-ER $\alpha$ together with GFP-tagged wild-type PES1 or K517R for 24 h. The cells were starved for 3 days, and then treated with or without $10 \mathrm{nM} \mathrm{E2} \mathrm{for} 24 \mathrm{~h}$. The cell extract was subjected to western blot with anti-Flag antibody. F. COS-7 cells were transfected with Flag-ER $\alpha$, GFP-tagged wild-type PES1 or K517R together with or without Flag-UBC9, and then subjected to western blot with anti-Flag antibody. 


\section{DISCUSSION}

Posttranslational modification of proteins endows proteins with multiple functions. SUMOylation, an important post-translation modification, manifests its effect through regulating protein-protein interaction, protein stability and protein localization. Although PES1 could be modified by SUMO-1, -2 and -3 , the modification was mainly carried out by SUMO-1 (Figure 1), and the modification resulted in improved stability for PES1 by inhibiting its ubiquitination (Figure 3). PES1 is a nuclear protein that is involved in the synthesis and maturation of ribosome. It also has an effect on chromatin stretch. Recent studies have demonstrated that PES1 can promote the development of different tumors [7, 9, 15]. We observed enhancement of ER $\alpha$ stability induced by PES1 SUMOylation, whether with or without E2 treatment, which led to the transactivation of ER $\alpha$ along with the growth of breast cancer cells in vivo and in vitro (Figure 5-7).
SUMOylation also regulates the stability of proteins. Consistent with this feature was the longer half-life of wild type PES1 compared to PES1 K517R (Figure 3B). Ubiquitinmediated proteolysis pathway exerts a crucial effect on many cellular physiological functions by regulating different proteins, such as cell surface receptors, transcriptional factors and cofactors. In this study, we showed that PES1 was targeted by ubiquitin and the ubiquitinated level of wild-type PES1 was lower than that of the mutant (Figure 3C). One possible reason could be that PES1 SUMOylation inhibited the interaction between PES1 and the ubiquitin E3 ligase Trim 23, leading to repression of PES1 ubiquitination (Figure 4).

The conjugation of SUMO with a target protein often leads to a change in the conformation of the protein, which then influences its interaction with other proteins. Wildtype PES1 could interfere with the association of ER $\alpha$ with CHIP, while the mutant PES1 K517R almost had no effect. (Figure 5B and 5C). In the absence of E2, CHIP (ubiquitin E3 ligase) constantly targets ER $\alpha$ for its degradation,
A

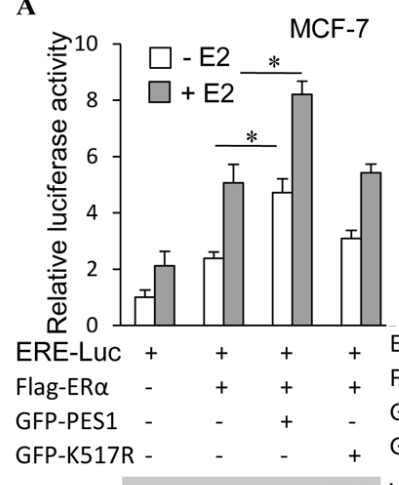

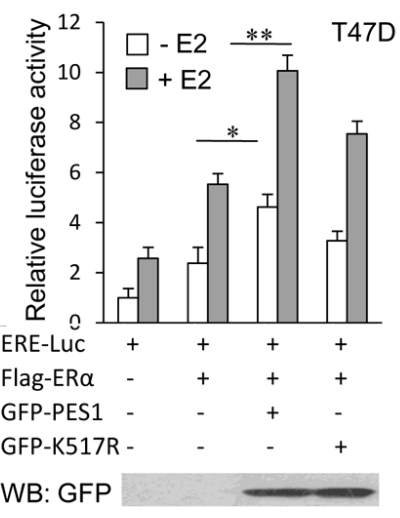
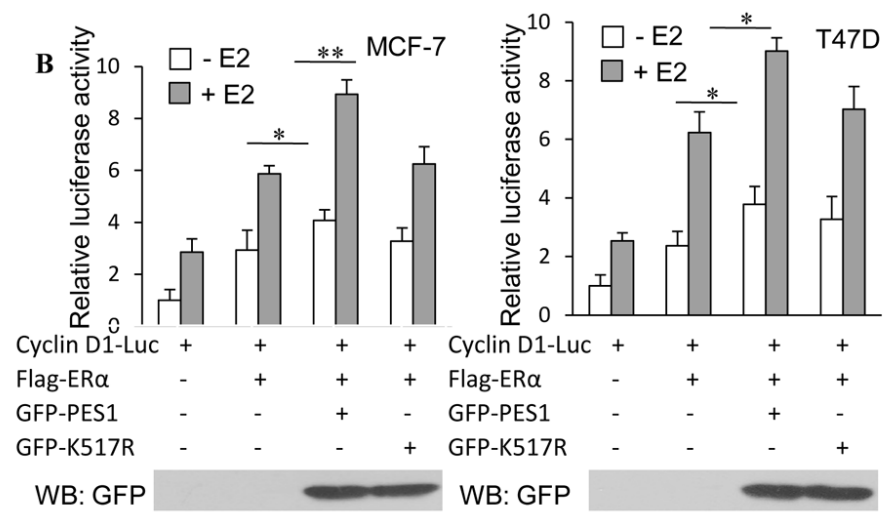
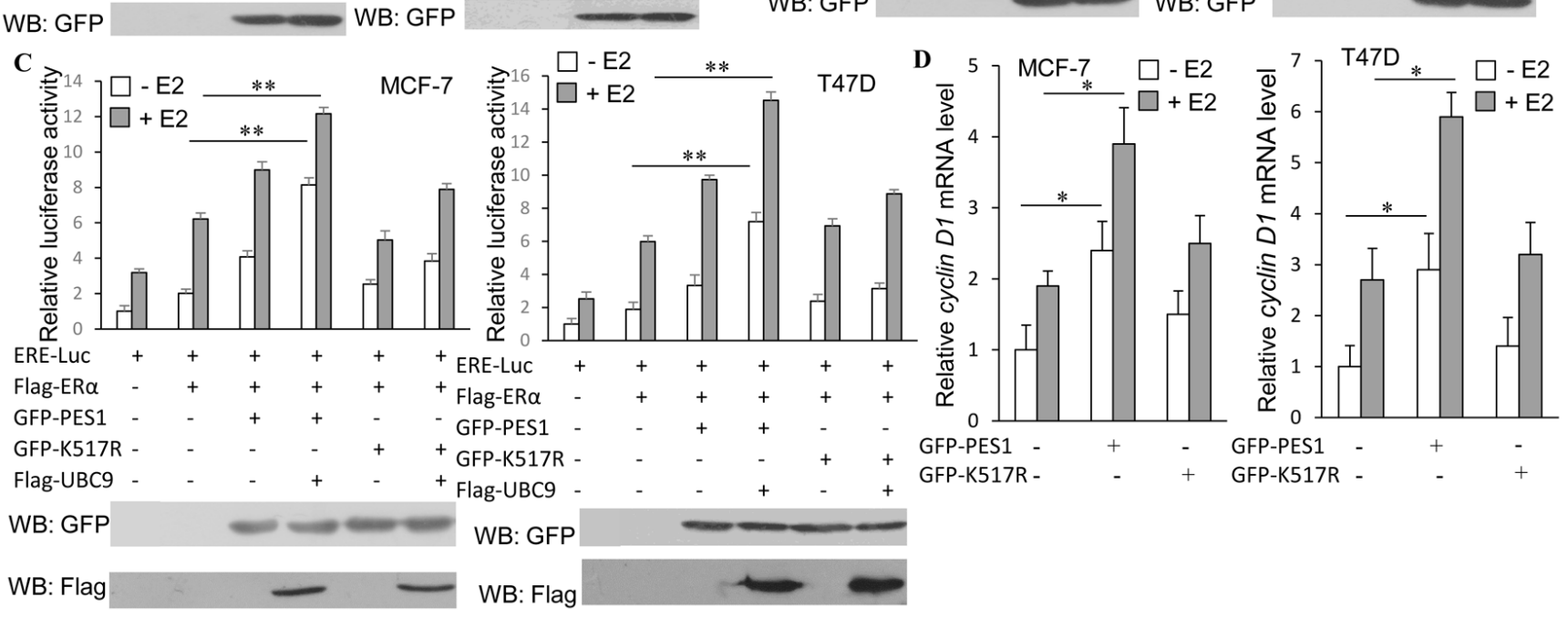

Figure 6: SUMOylation of PES1 upregulates the transcriptional activities of ER $\alpha$ and its downstream target gene. A. MCF-7 or T47D cells were transfected with ERE-Luc or with ER $\alpha$, or with ER $\alpha$ and GFP-tagged wild-type PES1 or K517R. Luciferase activity was measured either with or without pre-treatment of the cells with $10 \mathrm{nM}$ E2 for $16 \mathrm{~h}$. B. Similar experiment as described in A was performed, but the cells were transfected with Cyclin D1-luciferase. C. MCF-7 or T47D cells were transfected with ERE-Luc or with ER $\alpha$, or with ER $\alpha$ and GFP-tagged wild-type PES1 or K517R together with or without Flag-UBC9. Luciferase activity was measured either with or without pre-treatment of the cells with $10 \mathrm{nM} \mathrm{E2} \mathrm{for} 16 \mathrm{~h}$. D. MCF-7 or T47D cells were transfected with wild-type PES1 or K517R. The cells were pre-treated with or without $10 \mathrm{nM}$ E2 for $16 \mathrm{~h}$ and then subjected to RT-PCR to measure the mRNA level of Cyclin D1. The mRNA level of cyclin D1 was expressed relative to $G A P D H$ transcriptional level. '*', $p<0.05$; '**', $p<0.01$. 

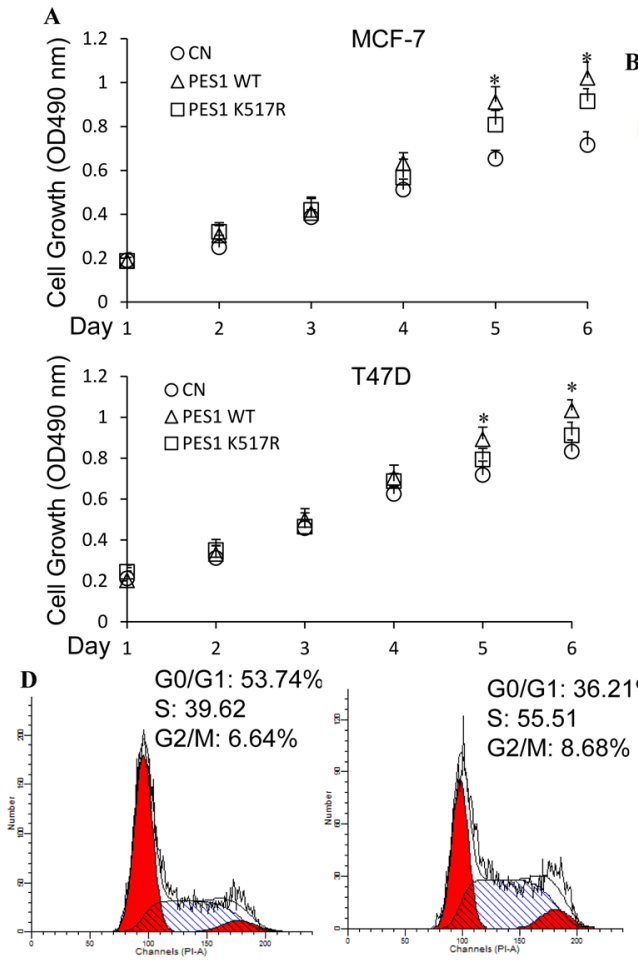

CN

PES1
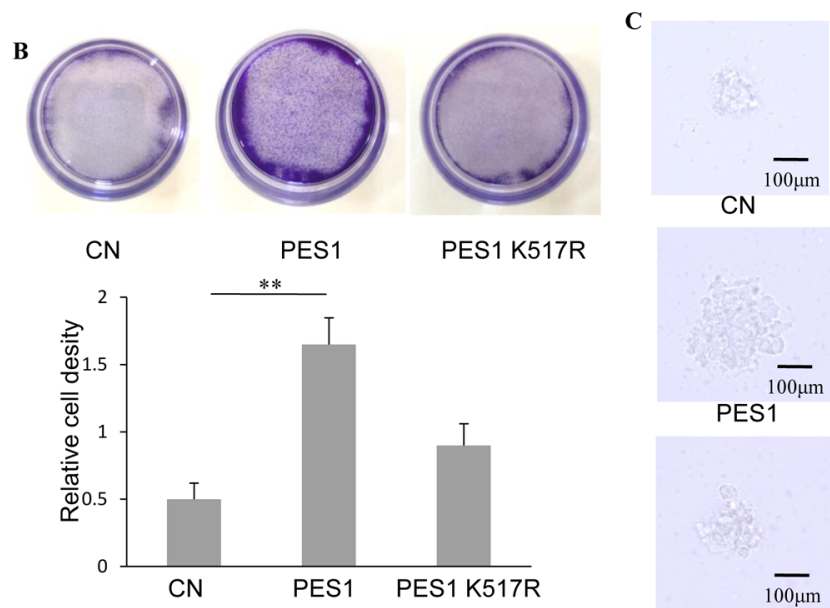

CN

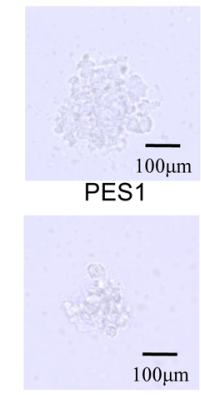

PES1 K517R

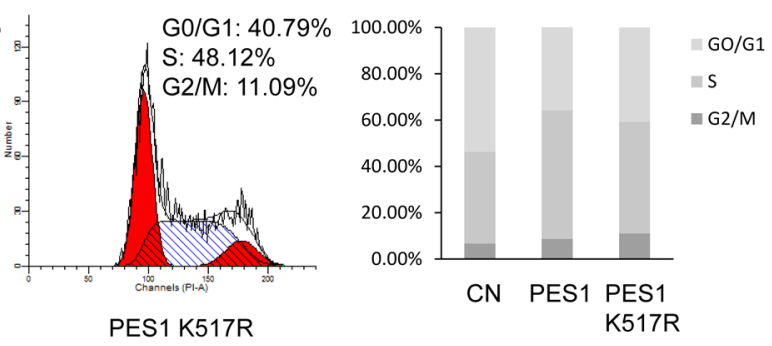

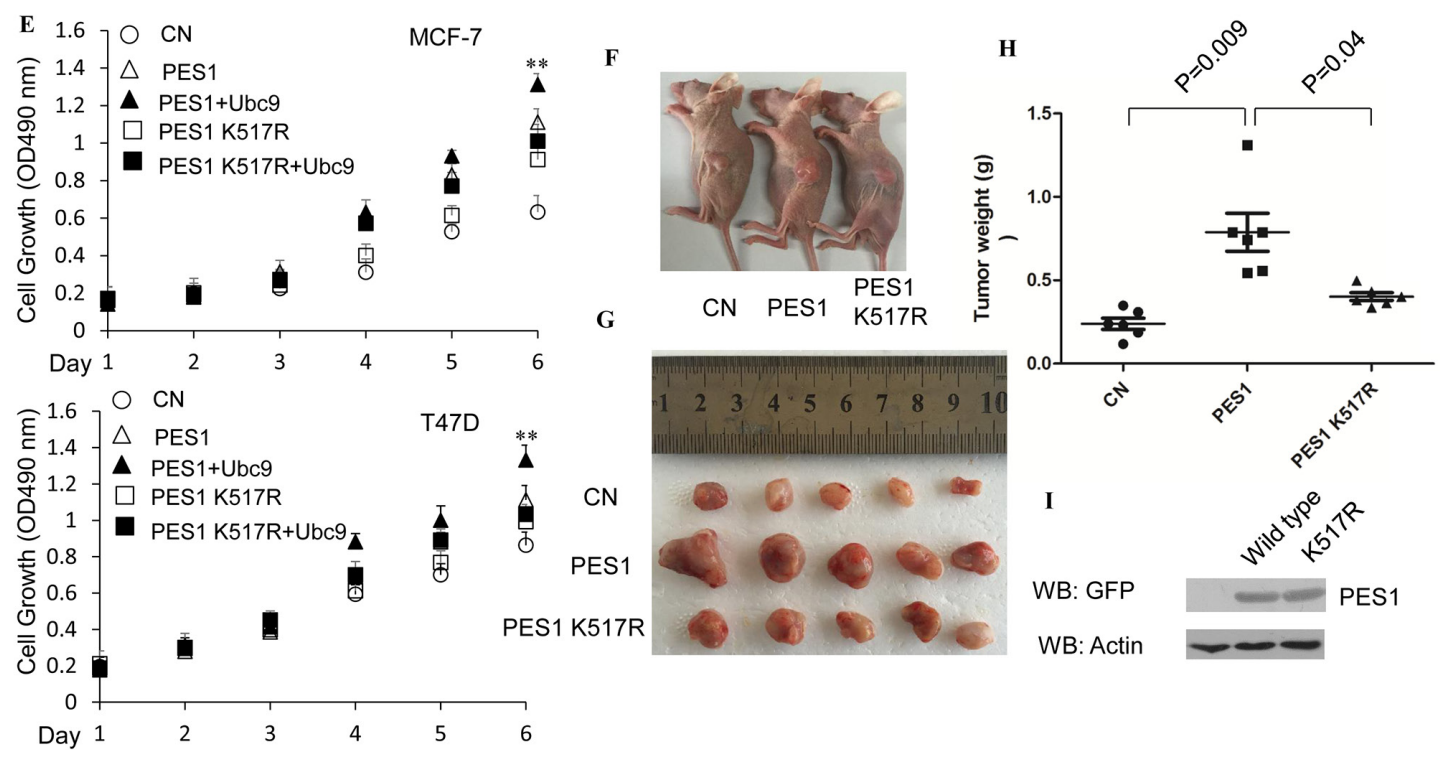

Figure 7: SUMOylation of PES1 promotes the growth of breast cancer cell lines. A. MCF-7 or T47D cells were stably transfected with GFP-tagged PES1 or K517R. The cells were grown in the presence of E2 and then subjected to MTT assay performed according to the manufacturer's instructions. B. Crystal violet staining (MCF-7 cells). The cells were treated with E2 for 7 days. Viable colonies were stained with $0.1 \%$ crystal violet and photographed (upper panel). Relative cell density (the lower panel) was obtained from three independent experiments. C. Representative colonies of each experimental group are shown. MCF-7 cells transfected with GFP-C1, GFP-PES1 or GFP-PES1 K517R were selected by growth in the presence of G418 (1 mg/ml) for 2 weeks. The cells were then used to prepare the soft agar colony culture. Photographs of the colonies were taken 10 days after seeding. All experiments were repeated at least three times. D. MCF-7 cells were transfected with GFP-C1, GFP tagged wild-type or K517R. Flow cytometry analysis was performed after $36 \mathrm{~h}$ of growth in the presence of $\mathrm{E} 2(10 \mathrm{nM})$. E. MCF-7 or T47D cells were stably transfected with GFP-tagged PES1 or K517R together with or without UBC9. The cells were grown in the presence of E2 and then subjected to MTT assay performed according to the manufacturer's instructions. F. Comparison of the sizes of tumors formed 30 days after the mice were injected with the tumor cells overexpressing GFP-C1, GFP-tagged PES1 or K517R. (G and H) Tumor images G. and tumor weight $\mathbf{H}$. were shown. 44 days after the mice were injected subcutaneously with MCF-7 cells overexpressing GFP-C1, GFP-tagged PES1 or K517R. n=5 mice per group in (F, G and H). I. The expression of GFP-tagged PES1 or K517R in the tumors extracted from the three groups of mice. '*', $p<0.05$; $^{\text {'**', }}, p<0.01$. 
and PES1 protects ER $\alpha$ from ubiquitination-mediated degradation via inhibiting its interaction with CHIP [9]. However, in the presence of E2, the level of ER $\alpha$ wouldbe reduced through an enhancement of its ubiquitination independent of ChIP [14]. Wild-type PES1 could enhance the stability of ER $\alpha$ with or without E2 treatment (Figure $5 \mathrm{~A}, 5 \mathrm{E}$ and $5 \mathrm{~F}$ ), while K517R lost this ability to protect $\mathrm{ER} \alpha$ from ubiquitination (Figure 5C). This showed that enhancement of ER $\alpha$ stability by PES1 was dependent on the SUMOylation status of PES1. In the E2-ER $\alpha$ signaling pathway, E2 plays important roles in maintaining the normal function of the mammary gland and it also functions as a potent mammary mitogen [16, 17]. E2 regulates gene transcription mainly through binding with $\mathrm{ER} \alpha$ to activate the transcription of ER $\alpha$-targeted genes, which are involved in cell growth. Enhancement in ER $\alpha$ stability is important to its function. However, E2 can also lead to the loss of ER $\alpha$ via degradation, but the lack of decrease in ER $\alpha$ level in the presence of E2 was a result of E2-stimulated PES1 SUMOylation that effectively maintained the stability of ER $\alpha$, thereby counteracting the loss of ER $\alpha$ by the direct action of E2. Many studies have tried to understand the effect of ER $\alpha$ on the generation and development of breast cancer, and aberrant ER $\alpha$ signaling is thought to be closely associated with the occurrence of ER $\alpha$ positive breast cancer $[18,19]$. Our findings may therefore provide yet a different view on the effect of E2 on ER $\alpha$-positive breast tumorgenesis. The activation of $\mathrm{ER} \alpha$ by E2 is a cycling process, in which E2 promotes the activity of ER $\alpha$, but at the same time, also promotes the degradation of ER $\alpha$ via increasing its ubiquitination independent of CHIP [14]. The end of the activation cycle is accompanied by the degradation of ER $\alpha$. In PES1-overexpressed cells, PES1 could rescue ER $\alpha$ from degradation mediated by E2 (Figure 5E, 5F) and this may presumably prolong the transactivation activity of $\mathrm{ER} \alpha$ in breast cancer cells. Previous research has shown that PES1 displays high expression in different tumors, including breast cancer [15]. So we proposed that in ER $\alpha$ positive breast cancer cell, SUMOylated PES1 may protect $\mathrm{ER} \alpha$ from ubiquitin degradation whether in the presence or absence of E2, and this protection would promote the growth of ER $\alpha$-positive breast cancer cells.

The development of cancer is usually caused by aberrant cell cycle. Cell cycle proteins Cyclin D1 controls the transition from the $\mathrm{G} 1$ phase to $\mathrm{S}$ phase, and it is commonly upregulated in breast cancer cells, leading to defects in mammary development and contributing to the generation of breast cancer, probably by promoting cell growth [20]. Wild-type PES1 promoted the expression of cyclin D1 in breast cancer cells, and the activation of cyclin D1 by PES1 was subject to regulation by SUMOylation, since the mutant PES1 that could not be SUMOylated had a weaker effect on the regulation of cyclin D1 expression (Figure 6). Previous studies have shown that overexpression of PES1 can lead to an increase in the percentage of 32D IRS- 1 cell in the G2/M phase [21], whereas knockdown of PES1 can shorten the G2/M phase for colon cancer cells as well as repressing the growth of xenografts [7]. This is consistent with our data, which showed that wild-type PES1 upregulated the expression of cyclin D1 through maintaining the stability of ER $\alpha$, and consequently, PES1 positively affected the cell cycle progress and the growth of breast cancer cells.

In summary, we demonstrated in this study that SUMOylation promoted the function and stability of PES1 and identified K517 as the key SUMOylation site. SUMOylation of PES1 also increased the stability of ER $\alpha$ by inhibiting the interaction between $\operatorname{ER} \alpha$ and its ubiquitin E3 ligase CHIP, resulting in the inhibition of ER $\alpha$ ubiquitination. The ultimate effect of PES1 SUMOylation at the cell level was the acceleration of cell proliferation and cell cycle progress, as demonstrated for two different breast cancer cell lines. This work has extended our understanding of the mechanism behind PES1-mediated regulation of transcription factors and the consequent promotion of breast cancer cell proliferation. However, much still needs to be explained, such as how PES1 SUMOylation can regulate the stability of ER $\alpha$ in the presence of E2, and whether the promotion of breast cancer cell growth by PES1 SUMOylation is a general phenomenon that also happens in other cancer cells.

\section{MATERIALS AND METHODS}

\section{Cell culture}

COS-7, MCF-7 and T47D cells have been used in our previous study [22, 23]. For the cell starvation assay, MCF-7 and T47D cells were cultured in medium containing $2 \%$ charcoal-stripped fetal bovine serum (Gibico) and without phenol red for several days, and then treated with or without E2 $(10 \mathrm{nM})$ for several hours.

\section{Plasmids, antibodies, si RNA and reagents}

Rabbit anti-Myc, anti-Flag, mouse anti-Flag (M2) and cycloheximide (CHX) were obtained from Sigma. Rabbit GFP was purchased from GeneTex. Rabbit antiPES1 and 17 $\beta$-estrogen (E2) were obtained from Abcam. Rabbit anti-ER $\alpha$ was obtained from Millipore. MG132 was purchased from Merk. The siRNA sequences, siTrim 23 and si control, were obtained from GenePharma RNAi Company and their sequences are shown in Supplementary Table S1.

HA-PES1 was obtained from Dr. Dirk Eick (Institute of Clinical Molecular Biology and Tumor Genetics, GSF Research Center, Germany). GFP-PES1 was constructed in our laboratory. The PES1 mutants GFP-K249R, K517R, and 2KR were prepared using the QuikChange Site-Directed Mutagenesis Kit (Stratagene, La Jolla, CA) using HA-PES1 as the template. Myc-tagged SUMO-1, SUMO-2, SUMO-3 
along with GFP-SUMO1 and its mutant GFP-SUMO1/GA have been used in our previous studies [11, 12]. Flag-UBC9 was amplified by PCR and subcloned into Flag-pCDNA3.1 vector at $X h o 1$ and $E c o$ R 1 sites.

\section{Immunoprecipitation and western blotting}

Cells harvested from the appropriate culture were lysed and the soluble fraction of the cell extract was subjected to western blot and immunoprecipitation were conducted as previously described $[11,24]$. Clean Blot IP Detection Reagent was purchased from Thermo Scientific.

\section{Immunofluorescence staining}

MCF-7 cells were transfected with GFP-tagged wild-type or mutant PES1 K517R for $36 \mathrm{~h}$, and the cells were then subjected to immunofluorescence staining assay as previously described [11].

\section{In vitro SUMOylation assay}

The vitro SUMOylation assay was performed using a SUMOylation Kit purchased from Enzo Life Sciences (NY, USA). In brief, purified GST-PES1 was incubated in a mixture containing recombinant E1, Ubc9 (E2), and Mg-ATP with or without SUMO-1 for $1 \mathrm{~h}$ at $30^{\circ} \mathrm{C}$ according to the manufacturer's instructions. The reaction was terminated with SDS-PAGE loading buffer.

\section{Luciferase reporter assay}

MCF-7 and T47D cells transfected with the appropriate plasmids for $24 \mathrm{~h}$ were starved for 2 days followed by stimulation with or without E2 $(10 \mathrm{nM})$ for another $16 \mathrm{~h}$. The cells were then subjected to luciferase assay using a dual luciferase Kit (Promega, USA).

\section{RNA extract and reverse transcription-PCR}

MCF-7 cells were stimulated with or without E2 $(10 \mathrm{nM})$ for $16 \mathrm{~h}$ after they had been starved for 2 days. Total RNA was extracted as previously described [22]. The primers used for the RT-PCR analysis of cyclin D1 and $G A P D H$ were as previously described [22]. Cyclin $D 1$ mRNA level was normalized with the GAPDH mRNA level.

\section{Cell proliferation assays}

MCF-7 and T47D cells were stably transfected with GFP-C1, GPF-PES1 or GFP-K517R. The cells were starved for 1 day, and then stimulated with E2 (10 nM) for several days. MTT assay was performed using an MTT assay kit obtained from KeyGEN BioTECH (Nanjing, China). For cell cycle analysis, MCF-7 cells transfected with GFP-C1, GPF-PES1 or GFP- K517R were cultured in the presence of E2 (10nM) for $36 \mathrm{~h}$, and then subjecting to flow cytometry analysis according to the protocol as previously described [22]. For crystal violet staining assay, MCF-7 cells transfected with GFP-C1, GPF-PES1 or GFPPES1 K517R were maintained in DMEM (containing 10\% fetal bovine serum) medium containing G418 (1 mg/ml) for 2 weeks. About $5 \times 10^{3}$ cells/well were then plated in 6 -well plate and grown in the presence of E2 (10 nM) for 7 days. After that the cells were subjected to crystal violet staining according to a previous report [23].

\section{Soft-agar colony culture}

MCF-7 cells were transfected with wild-type PES1, K517R or GFP-C1 and grown in DMEM (containing 10\% fetal bovine serum) medium containing G418 (1 mg/ml) for 2 weeks. The cell suspension containing 2000 cells/ group were used for soft-agar colony culture assay as previously described $[25,26]$.

\section{Human breast cancer xenograft model}

About $1 \times 10^{7} \mathrm{MCF}-7$ cells stably transfected with GFP-PES1, GFP-PES1 K517R or GFP-C1 were resuspended in $50 \%$ Corning $\AA$ Matrigel $($ Basement Membrane Matrix High Concentration (Corning) in a final volume of $100 \mu \mathrm{l}$, and then injected into the left flank of five to six week-old female athymic nude mice (BALB/c mice, from Animal Experiment Center of Dalian Medical University). All mice were maintained under specific pathogen-free (SPF) conditions. All experiments involving animals were performed under the regulations set by the Ethics Committee for Dalian University of Technology. Tumor growth was facilitated by feeding the animals with water containing E2 at a concentration of $1 \mathrm{mg} / \mathrm{L}$ [27]. After 44 days, the mice were killed in a humane manner as previously described [28].

\section{Statistical analysis}

All statistical analyses of data were performed with ANOVA. Data were given as means \pm SDs, and statistical significance was considered at either $P$ value $<0.05$ or 0.01 level.

\section{ACKNOWLEDGMENTS}

We are grateful to Dr. Dirk Eick (Institute of Clinical Molecular Biology and Tumor Genetics, GSF Research Center, Germany) for the present of HA-PES1. We thank Dr. Jorma J. Palvimo (University of Kuopio) for giving us GFP-SUMO1 and its mutant GFP-SUMO1/ GA (Cambridge Research Institute). We are grateful to Dr. Carolyn L. Smith (Baylor College of Medicine) for the present of pcDNA3.1/ERE luciferase reporter. We thank Dr. Paul D. Sadowaki (University of Toronto, Canada) 
for giving us Myc-tagged SUMO1, SUMO2 and SUMO3 plasmids. We are grateful to Dr. Alan K. Chang (Liaoning University) for helpful discussion and for revising the language of the manuscript.

\section{CONFLICTS OF INTEREST}

All the authors have declared that no competing interests exist.

\section{GRANT SUPPORT}

This research was supported by grants ( 31271500 to H.W., 31301159 to S.L. and 81301504 to M.W.) from National Nature Science Foundation of China and Program for Liaoning Innovative Research Team in University (LT2015008 to H.W.). The funders had no roles in study design, data collection and analysis, decision to publish, or prepare of the manuscript.

\section{REFERENCES}

1. Haffter $P$, Granato $M$, Brand $M$, Mullins MC, Hammerschmidt M, Kane DA, Odenthal J, van Eeden FJ, Jiang YJ, Heisenberg CP, Kelsh RN, Furutani-Seiki M, Vogelsang E, et al. The identification of genes with unique and essential functions in the development of the zebrafish, Danio rerio. Development. 1996; 123:1-36.

2. Driever W, Solnica-Krezel L, Schier AF, Neuhauss SC, Malicki J, Stemple DL, Stainier DY, Zwartkruis F, Abdelilah S, Rangini Z, Belak J and Boggs C. A genetic screen for mutations affecting embryogenesis in zebrafish. Development. 1996; 123:37-46.

3. Dunham I, Shimizu N, Roe BA, Chissoe S, Hunt AR, Collins JE, Bruskiewich R, Beare DM, Clamp M, Smink LJ, Ainscough R, Almeida JP, Babbage A, et al. The DNA sequence of human chromosome 22. Nature. 1999; 402:489-495.

4. Grimm T, Holzel M, Rohrmoser M, Harasim T, Malamoussi A, Gruber-Eber A, Kremmer E and Eick D. Dominantnegative Pes1 mutants inhibit ribosomal RNA processing and cell proliferation via incorporation into the PeBoWcomplex. Nucleic Acids Res. 2006; 34:3030-3043.

5. Nakaguro M, Kiyonari S, Kishida S, Cao D, MurakamiTonami Y, Ichikawa H, Takeuchi I, Nakamura S and Kadomatsu K. Nucleolar protein PES1 is a marker of neuroblastoma outcome and is associated with neuroblastoma differentiation. Cancer science. 2015; 106:237-243.

6. Kinoshita Y, Jarell AD, Flaman JM, Foltz G, Schuster J, Sopher BL, Irvin DK, Kanning K, Kornblum HI, Nelson PS, Hieter P and Morrison RS. Pescadillo, a novel cell cycle regulatory protein abnormally expressed in malignant cells. J Biol Chem. 2001; 276:6656-6665.
7. Xie W, Feng Q, Su Y, Dong B, Wu J, Meng L, Qu L and Shou C. Transcriptional regulation of PES1 expression by c-Jun in colon cancer. PLoS One. 2012;7:e42253.

8. Gaspar I and Szabad J. Glu415 in the alpha-tubulins plays a key role in stabilizing the microtubule-ADP-kinesin complexes. J Cell Sci. 2009; 122:2857-2865.

9. Cheng L, Li J, Han Y, Lin J, Niu C, Zhou Z, Yuan B, Huang K, Jiang K, Zhang H, Ding L, Xu X and Ye Q. PES1 promotes breast cancer by differentially regulating ERalpha and ERbeta. J Clin Invest. 2012; 122:2857-2870.

10. Guo C and Henley JM. Wrestling with stress: roles of protein SUMOylation and deSUMOylation in cell stress response. IUBMB Life. 2014; 66:71-77.

11. Hong Y, Xing X, Li S, Bi H, Yang C, Zhao F, Liu Y, Ao $\mathrm{X}$, Chang $\mathrm{AK}$ and $\mathrm{Wu} \mathrm{H}$. SUMOylation of $\mathrm{DEC} 1$ protein regulates its transcriptional activity and enhances its stability. PLoS One. 2011; 6:e23046.

12. Bi H, Li S, Wang M, Jia Z, Chang AK, Pang $P$ and $W u H$. SUMOylation of GPS2 protein regulates its transcriptionsuppressing function. Molecular biology of the cell. 2014; 25:2499-2508.

13. Maiorana A, Tu X, Cheng G and Baserga R. Role of pescadillo in the transformation and immortalization of mammalian cells. Oncogene. 2004; 23:7116-7124.

14. Tateishi Y, Kawabe Y, Chiba T, Murata S, Ichikawa K, Murayama A, Tanaka K, Baba T, Kato S and Yanagisawa J. Ligand-dependent switching of ubiquitin-proteasome pathways for estrogen receptor. EMBO J. 2004; 23:4813-4823.

15. Li J, Yu L, Zhang H, Wu J, Yuan J, Li X and Li M. Down-regulation of pescadillo inhibits proliferation and tumorigenicity of breast cancer cells. Cancer Sci. 2009; 100:2255-2260.

16. Sternlicht MD. Key stages in mammary gland development: the cues that regulate ductal branching morphogenesis. Breast Cancer Res. 2006;8:201.

17. Yager JD and Davidson NE. Estrogen carcinogenesis in breast cancer. N Engl J Med. 2006; 354:270-282.

18. Soysal SD, Kilic IB, Regenbrecht CR, Schneider S, Muenst S, Kilic N, Guth U, Dietel M, Terracciano LM and Kilic E. Status of estrogen receptor 1 (ESR1) gene in mastopathy predicts subsequent development of breast cancer. Breast cancer research and treatment. 2015; 151:709-715.

19. Mauro L, Pellegrino M, Giordano F, Ricchio E, Rizza P, De Amicis F, Catalano S, Bonofiglio D, Panno ML and Ando S. Estrogen receptor-alpha drives adiponectin effects on cyclin D1 expression in breast cancer cells. FASEB journal. 2015; 29:2150-2160.

20. Caldon CE, Sutherland RL and Musgrove E. Cell cycle proteins in epithelial cell differentiation: implications for breast cancer. Cell Cycle. 2010;9:1918-1928.

21. Prisco M, Maiorana A, Guerzoni C, Calin G, Calabretta B, Voit $\mathrm{R}$, Grummt I and Baserga R. Role of pescadillo and upstream 
binding factor in the proliferation and differentiation of murine myeloid cells. Mol Cell Biol. 2004; 24:5421-5433.

22. Li S, Wang M, Ao X, Chang AK, Yang C, Zhao F, Bi H, Liu Y, Xiao L and Wu H. CLOCK is a substrate of SUMO and sumoylation of CLOCK upregulates the transcriptional activity of estrogen receptor-alpha. Oncogene. 2013; 32:4883-4891.

23. Xiao L, Chang AK, Zang MX, Bi H, Li S, Wang M, Xing X and $\mathrm{Wu} \mathrm{H}$. Induction of the CLOCK gene by E2-ERalpha signaling promotes the proliferation of breast cancer cells. PLoS One. 2014; 9:e95878.

24. Cascone I, Napione L, Maniero F, Serini G and Bussolino F. Stable interaction between alpha5beta1 integrin and Tie2 tyrosine kinase receptor regulates endothelial cell response to Ang-1. The Journal of cell biology. 2005; 170:993-1004.

25. Bi H, Li S, Qu X, Wang M, Bai X, Xu Z, Ao X, Jia Z, Jiang $X$, Yang $Y$ and Wu H. DEC1 regulates breast cancer cell proliferation by stabilizing cyclin E protein and delays the progression of cell cycle S phase. Cell death \& disease. 2015; 6:e1891.

26. Li YY, Bao YL, Song ZB, Sun LG, Wu P, Zhang Y, Fan C, Huang YX, Wu Y, Yu CL, Sun Y, Zheng LH, Wang GN and Li YX. The threonine protease activity of testes-specific protease 50 (TSP50) is essential for its function in cell proliferation. PLoS One. 2012; 7:e35030.

27. Lee ES, Na K and Bae YH. Doxorubicin loaded $\mathrm{pH}$-sensitive polymeric micelles for reversal of resistant MCF-7 tumor. Journal of controlled release. 2005; 103:405-418.

28. Lombardo Y, Filipovic A, Molyneux G, Periyasamy M, Giamas G, Hu Y, Trivedi PS, Wang J, Yague E, Michel L and Coombes RC. Nicastrin regulates breast cancer stem cell properties and tumor growth in vitro and in vivo. Proceedings of the National Academy of Sciences of the United States of America. 2012; 109:16558-16563. 